\title{
DIE ÜBERLIEFERUNG DER MOTETTE SURGE, PROPERA, AMICA MEA VON JACOB HANDL - GALLUS UND IHRE BEARBEITUNGEN VON SIXTUS KARGEL UND BLASIUS AMON: EIN BEITRAG ZUR GALLUS-REZEPTION
}

\author{
MARKO MOTNIK \\ Universität für Musik und darstellende Kunst Wien, Universität Wien
}

Izvleček: Prispevek se ukvarja z razširjenostjo štiriglasnega moteta Surge, propera, amica mea Jacobusa Handla - Gallusa. Jedro razprave predstavljata doslej $v$ strokovnih krogih komaj opaženi predelavi te skladbe Sixta Kargla in Blasiusa Amona. Obe deli sta nastali pred natisom Gallusovega moteta.

Ključne besede: Jacobus Handl - Gallus, Surge, propera, amica mea, Sixtus Kargel, intabulacija za lutnjo, Blasius Amon, Missa super Surge propera.
Abstract: In the present article, the transmission history of the four-voice motet Surge, propera, amica mea by Jacob Handl Gallus is discussed. Little noted in the scholarly literature, the transcriptions by Sixtus Kargel and Blasius Amon, which predate the appearance of this motet in print, stand at the centre of this discussion.

Keywords: Jacob Handl Gallus, Surge, propera, amica mea, Sixtus Kargel, lute intabulation, Blasius Amon, Missa super Surge propera.

Dass zumindest einige Werke von Gallus mehrere Jahre vor ihrer Drucklegung entstanden sind, lässt sich aus verschiedenen Beobachtungen zur Überlieferungssituation ableiten. Vier Messen des Komponisten, denen seine eigenen Motetten als Modell dienten, nämlich Missa ad imitationem Pater noster, Missa ad imitationem Elisabethae impletum est, Missa super Elisabeth Zachariae und Missa super Locutus est Dominus ad Moysen publizierte der Komponist im Jahr 1580, ${ }^{1}$ während ihre Vorlagen erst zwischen 1586 und 1590 veröffentlicht wurden. ${ }^{2}$ Tomasz Jeż machte in seinen Studien ebenso auf die handschriftlichen Quellen schlesischer Provenienz aufmerksam, die etliche Werke von Jacob Handl Gallus enthalten und noch vor der Drucklegung der einbezogenen Kompositionen niedergeschrieben wurden. ${ }^{3}$

1 Iacobus Handl, Missarum VII. \& VIII. vocum, liber I. SELECTIORES QVAEDAM MISSAE, [...], Praga, G. Nigrinus, 1580 (RISM A/I: H 1976); sowie ders. Missarum VI. vocum, liber I. SELECTIORES QVAEDAM MISSAE, [...], Praga, G. Nigrinus, 1580 (RISM A/I: H 1977).

2 Die Motetten Pater noster und Locutos est Dominus sind im Jahr 1586, Elisabethae [vero] impletum est 1587 und das Werk Elisabeth Zachariae im Jahr 1590 erschienen.

3 Vgl. Tomasz Jeż, The motets of Jacob Handl in inter-confessional Silesian liturgical practice, im vorliegenden Heft De musica disserenda, S. 35-46; sowie ders. Twórczość Jakoba Handla 
Zugleich zeugen die Lautenintavolierung der Motette Surge, propera von Sixtus Kargel (1586) und die Missa super Surge propera von Blasius Amon (1588) von einer frühen handschriftlichen Verbreitung eines Werkes von Gallus und dies weit über die Grenzen seines Wirkungsbereichs hinaus.

Die vierstimmige Motette Surge, propera, amica mea von Jacob Handl Gallus erschien $1590 \mathrm{im}$ QVARTVS TOMUS MVSICI OPERIS (RISM A/I: H 1985). Diesen letzten und umfangreichsten Teil der Motettensammlung, der mit einem kaiserlichen Privileg in Prag bei Georg Nigrin in acht Stimmheften aufgelegt wurde, hatte Gallus für die Heiligenfeste bestimmt. Das Werk ist Ambrosius, dem Abt des Prämonstratenserklosters Zábrdovice (Obrowitz) gewidmet und die Dedikation ist auf den 1. Januar 1591 datiert. Es wird angenommen, dass das Werk dessen ungeachtet noch im Jahr 1590 gedruckt wurde. Gallus wollte wahrscheinlich mit der Widmung des zweiten Todestages des ihm nahe stehenden Abtes Caspar Schönauer, des Vorgängers von Ambrosius, gedenken.

Im Jahr 1590 druckte auch Adam Berg in München ein von Stephan Schormann herausgegebenes Sammelwerk mit dem Titel Suavissimorum modulorum selectissimae cantiones sacrae [...] (RISM A/I: 1590/6). An vierter Stelle ist darin die Motette Surge, propera enthalten; wie die übrigen Werke ohne Angabe des Autors. Außerdem schließt die Sammlung auch die Motette Elizabeth Zachariae (Nr. 23) und Missa super Elizabeth Zachariae (Nr. 24) von Gallus mit ein. Die kunstvolle Ordinariumsvertonung wird sogar am Titelblatt erwähnt: „His adjucta est missa eleganti artificio composita [...]. ${ }^{64} \mathrm{Die}$ sechsstimmige Motette Elizabeth Zacharie ist ebenfalls erst im vierten Teil von Opus musicum 1590 erschienen und wurde, wie erwähnt, bereits zehn Jahre davor von Gallus in der Messe verarbeitet und 1580 im zweiten Buch der Messen veröffentlicht. Da der Münchner Sammeldruck auf den 1. März 1590 datiert ist und der vierte Teil des Opus musicum wahrscheinlich erst gegen Ende des Jahres erscheinen sollte, griff Schormann zumindest bei der Herausgabe der zwei Motetten sicherlich auf die handschriftlichen Quellen und nicht auf den Druck zurück.

Der vierte Teil des Opus musicum bezieht neben der vierstimmigen Fassung von Surge,propera, amica mea (Nr. 54) auch eine doppelchörige Parallelvertonung des Textes zu acht Stimmen (Nr. 1) ein. Während die vierstimmige Motette in zwei Teilen die Verse 10 bis 14 des zweiten Hohenliedes umfasst, beschränkt sich das achtstimmige Werk auf die Verse 13 und 14 und entspricht somit der secunda pars der vierstimmigen Fassung. In den beiden für die Marienfeste bestimmten Werken wird von Gallus ein ähnliches, zum Teil identisches motivisches Material verwendet und bearbeitet.

In der vierstimmigen Motette charakterisiert Gallus in der Regel jeden neuen Vers auch mit einem neuen sogetto. Die Anfänge der Verse werden mit einem sukzessiven Eintritt der Stimmen eingeleitet, kurz nach dem imitatorischen Beginn ist jedoch eine für den Komponisten charakteristische Tendenz zur Homophonie zu konstatieren. Ähnlich wie in zahlreichen anderen vierstimmigen Werken von Gallus beobachten wir auch hier

v žródłach proweniencji Śląskiej, Muzyka, Kwartalnik Instytutu Sztuki Polskiej Akademii Nauk, Bd. 4, Warszawa, Instytut Sztuki, Polska Akademia Nauk, 2004, S. 27-62.

4 Suavissimorum Modulorum Selectissimae Cantiones Sacrae, München, 1590, Titelblatt, Exemplar der Staats- und Stadtbibliothek Augsburg, Mikrofiche Ausgabe, Erlangen, Harald Fischer Verlag, 1999. 
stellenweise eine Vorliebe für die Führung der Stimmen in Paaren. Das sogetto, das den Charakter der Motette am stärksten prägt, ist der aufsteigende Quintsprung im ersten Vers der prima pars. Das Motiv findet sein Gegenstück im Quintfall des ersten Verses der secunda pars, das den auffordernden Ausruf „Surge“ geradezu negiert. Ein weiteres Charakteristikum des Werkes ist die in zahlreichen Zusammenhängen auftretende stufenweise absteigende Quarte (z. B. Vers 2 in der prima, oder Vers 5 in der secunda pars). ${ }^{5}$

Die im Lautenbuch von Sixtus Kargel enthaltene Intavolierung der Motette Surge, propera wurde bereits 1901 im Biographisch-Bibliographischen Quellen-Lexikon von Robert Eitner im Abschnitt über Jakob Handl ${ }^{6}$ und 1905 in der Bibliographie der Werke von Gallus von Josef Mantuani aufgelistet. ${ }^{7}$ Das Werk wurde bisher allerdings, abgesehen von einigen flüchtigen Erwähnungen in der Fachliteratur, nicht näher untersucht.

Die biographischen Daten von Sixtus Kargel sind derart lückenhaft überliefert, dass keinerlei Vermutungen und Hypothesen aufgestellt werden können, auf welchem Weg er mit der Komposition von Gallus in Berührung kam. Die Herkunft des seinerzeit berühmten Lautenisten liegt im Dunkeln. Jedenfalls ist Kargel vor 1545 geboren, hielt sich um 1569 vermutlich in Mainz auf und arbeitete später als Herausgeber in der Druckwerkstatt von Bernhard Jobin in Strassburg. In den Jahren 1569 bis 1586 machte er mit einer Reihe gedruckter Bücher für Laute und Zither auf sich aufmerksam, deren Großteil heute verschollen ist. ${ }^{8}$ Spätestens seit 1574 stand Kargel als Lautenist im Dienst des Erzbischofs Johann von Manderscheid-Blankenheim, des Landgrafen von Elsass. ${ }^{9}$ Ähnlich wie Melchior Neusiedler war Sixtus Kargel zunächst einem virtuosen Lautenstil verpflichtet und von der italienischen Diminutionspraxis beeinflusst. In seiner letzten und umfangreichsten Sammlung von 1586, also auch in der Intavolierung von Surge, propera, ist dagegen geradezu eine Zurückhaltung in der Anwendung des Verzierungswerks zu beobachten.

Das Lautenbuch / viler Newerlesenen fleissiger / schöner Lautenstück von artlichen Fantaseien / künstlichen Musicartlichen Lateinischen Muteten / mit fünff vnd sechs stimmen allerhand lieblichen Teutschen / Frantzösischen vnd Italienischen Liedern / auch lustigen Passomezzen [...] ist 1586 bei Bernhard Jobin in Strassburg erschienen (RISM A/I: 1586/23). Die Widmung an den Bischof zu Strassburg ist auf den 2. September des Jahres datiert. Es handelt sich um die einzige Publikation von Kargel in deutscher Lauten-

5 Vgl. Iacobus Gallus, Opus Musicum IV/1: Harmoniae octo vocum, hrsg. von Edo Škulj, Monumenta artis musicae Sloveniae XIV, Ljubljana, ZRC SAZU, 1989, S. 1-6; sowie Iacobus Gallus, Opus Musicum IV/4: Harmoniae quatuor vocum, hrsg. von Edo Škulj, Monumenta artis musicae Sloveniae XVII, Ljubljana, ZRC SAZU, 1990, S. 5-11.

${ }^{6}$ Robert Eitner, Biographisch-Bibliographisches Quellen-Lexikon der Musiker und Musikgelehrten christlichen Zeitrechnung bis zur Mitte des neunzehnten Jahrhunderts, Bd. 5, Leipzig, Breitkopf \& Härtel, 1901, S. 15.

7 Josef Mantuani, Bibliographie der Werke von Gallus, Jacob Handl (Gallus), Opus musicum II, hrsg. von Emil Bezecny und Joseph Mantuani, Denkmäler der Tonkunst in Österreich 12, Wien, Artaria, 1905, S. XI.

8 Siehe Publikationen von Sixtus Kargel, aufgelistet in Howard Mayer Brown, Instrumental Music Printed Before 1600, A Bibliography, Cambridge, London, Harvard University Press, 1965, S. 239-344.

9 Vgl. Christian Bettels, Kargel, Sixtus, Die Musik in Geschichte und Gegenwart. Personenteil 9, 2. Auflage, Kassel [...], Bärenreiter, 2003, Sp. 1498-1499. 
tabulatur und ist laut Autor hauptsächlich für diejenigen bestimmt, die „der Italienischen und Frantzösischen Tabulaturn unerfahren“ sind. Sie sei ferner ,auffs verständlichst und richtigst zusammengetragen und geordnet" worden. ${ }^{10}$ Die Sammlung enthält außer sechs eigenständig komponierten Fantasien und einigen Tanzsätzen hauptsächlich intavolierte Werke verschiedener Komponisten (überwiegend von Orlando di Lasso), darunter als Nr. 25 ,SVRGE PROPEra, 4. vocum. [...] Iacobo Gallo.“ Das Werk kann auf einer sechssaitigen Laute realisiert werden.

\section{Notenbeispiel 1}

Sixtus Kargel, Intavolierung SVRGE PROPEra, 4. vocum, Beginn, T. 1-4 (oben) im Vergleich

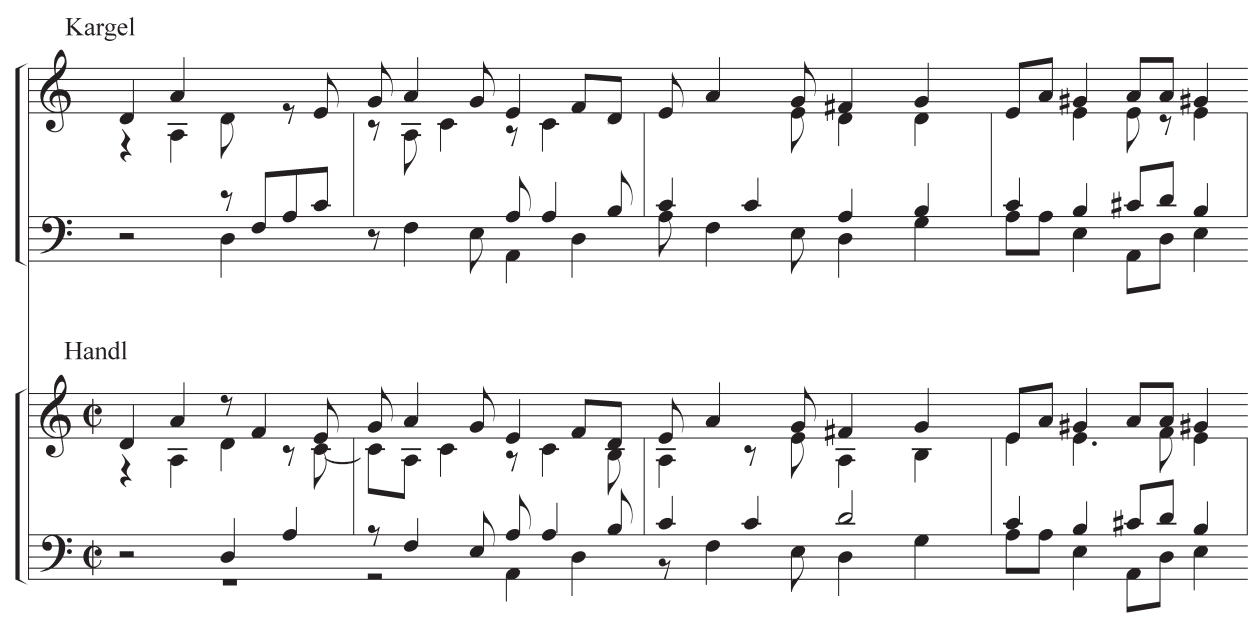

mit Jacob Handl, Surge, propera, amica mea (unten). ${ }^{11}$

Das Arrangement einer sprachgebundenen Vorlagenkomposition emanzipiert sich als ein eigenständiges Instrumentalwerk am auffälligsten in der Hinzufügung von instrumentalen Spielformeln, also in der Figuration. Diese sind in der Lautenfassung Surge propera selten und schwach ausgeprägt. Der Beitrag Kargels ist in den zur Vorlage hinzugefügten Spielfiguren und etwaigen Bearbeitungen der Textur dermaßen gering, dass der Anspruch einer lautenidiomatischen Fassung kaum erfüllt wird. Einige wenige ausgeschriebene Diminutionen sind lediglich in den Kadenzen zu finden, beziehungsweise an jenen Stellen, an denen die Satzstruktur der Vorlage weniger dicht ist (siehe Notenbeispiel 2). Es handelt sich dabei allerdings um keine kunstvollen Wendungen, sondern bloß um kurze Umspielungen herkömmlicher Art. Im gesamten Werk werden lediglich acht kurze Stellen verziert, davon sechsmal in der prima pars.

\footnotetext{
${ }^{10}$ Sixtus Kargel, Lautenbuch / viler Newerlesenen fleissiger / schöner Lautenstück [...], Straßburg, Bernhard Jobin, 1586, Titelblatt.

${ }^{11}$ Transkribiert nach dem Exemplar der Österreichischen Nationalbibliothek, Musiksammlung, Signatur SA.76.A.26. Mus 31.
} 
Notenbeispiel 2

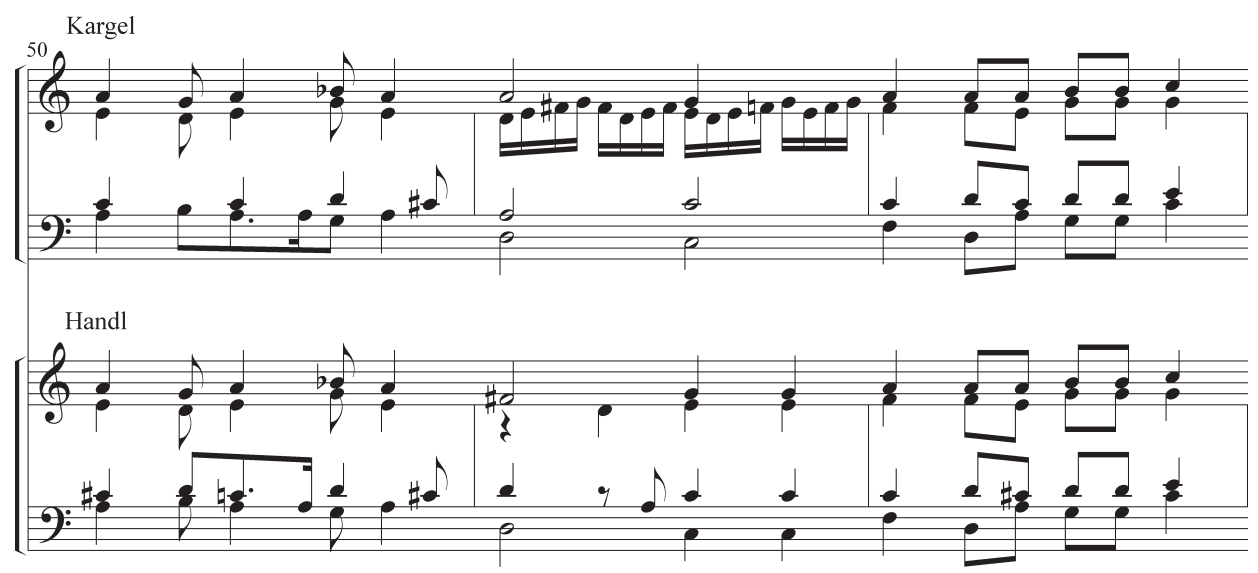

Sixtus Kargel, SVRGE PROPEra, T. 50-52

Die Unterschiede zur 1590 in Prag gedruckten Fassung sind in der Intavolierung trotzdem erheblich. Sie bestehen jedoch nicht im Gebrauch von etwaigen instrumentalen Mitteln, sondern in der hohen Fehlerhaftigkeit der Textur und eigenwilligen Setzung der Akzidentien. Wir beobachten zahlreiche willkürliche Veränderungen der Satzstruktur, Unklarheiten in den Rhythmuszeichen, ein Auslassen einer gesamten Mensur (Takt 12) oder auch unschlüssige Verschiebungen der Stimmen. Das Werk ist als äußerst fehlerhaft und korrupt zu bezeichnen. Seine Hauptproblematik kann in der Beharrung auf der relativ dichten Satzstruktur und ihrer Vierstimmigkeit gesehen werden. Indem die Stimmen nicht in ausreichendem Maße reduziert werden, entstand folglich kein Raum für die weiteren instrumentenidiomatischen Figuren und Wendungen. Da jedoch die ursprüngliche Abschrift der Motette, die dem Bearbeiter vorlag, nicht mehr zu eruieren ist, kann auch nicht beurteilt werden, welche Abweichungen von Kargel vorgenommen und welche gegebenenfalls auf eine fehlerhafte Quellenvorlage zurück zu führen sind. Obwohl die Lautenintavolierung aus musikalischer Sicht anspruchslos und künstlerisch unbefriedigend ist, bleibt die Bedeutung dieses Werkes als Zeugnis von Verbreitung und Rezeption der Werke von Jacob Handl Gallus nach wie vor ungeschmälert.

An dieser Stelle sollte noch kurz die Problematik der Akzidentien in den überlieferten Fassungen der Motette erläutert werden. In der Intavolierung von Kargel gibt es im Vergleich mit dem autorisierten Druck über 30 Abweichungen in der Bezeichnung der alterierten Töne. Eine wiederum unterschiedliche Tradierung der Akzidentien beobachten wir im oben genannten Sammeldruck Suavissimorum modulorum von Stephan Schormann. Es ist daher anzunehmen, dass in der ursprünglichen von Gallus niedergeschriebenen Quelle und in den frühen Abschriften der Motette wenige Alterationen eingetragen gewesen waren und die Fassungen von Kargel und Schormann nunmehr verschiedene zeitgenössische Aufführungspraktiken widerspiegeln. Umso strikter setzte Gallus die Alterationen im Druck von 1590, womöglich um die willkürlichen Lesarten und Interpretationen auszuschließen. 
Einem weitaus souveräneren Aneignungsprozess der Motette begegnen wir in der Missa super Surge propera von Blasius Amon. In der bis heute einzigen umfangreichen Studie über das Leben und Werk diesen österreichischen Komponisten konstatierte der Autor Caecilianus Huigens im Jahr 1914: „Viele Komponisten haben eine Motette ,Surge propera' wie Palestrina, Lassus, Gallus, Regnard, Praetorius, Merulo und andere. Die Motette, welche Amon benützt hat, habe ich nicht gefunden." ${ }^{2}$ Die Provenienz des Modells erkannte schließlich Walter Senn, der in den Veröffentlichungen des Tiroler Landesmuseums Ferdinandeum 1976 einen Beitrag zur Lebensgeschichte Amons veröffentlichte. ${ }^{13}$

Blasius Amon, um 1560 in Tirol geboren, wird ab 1568 als Sängerknabe in der Hofkapelle Erzherzogs Ferdinands II. in Innsbruck angeführt, die zeitweise der Leitung von Alexander Utendal unterlag. Im Jahr 1577 trat er in Brixen in den Franziskanerorden ein und blieb bis etwa 1580 im Franziskanerkloster in Innsbruck. Kurz danach ist ein Aufenthalt im Stift Zwettl belegt, das zu dieser Zeit von Abt Johannes Rueff geleitet wurde. Nach zwischenzeitlichen Studien in Venedig (etwa 1582 bis 1585) bekleidete Amon das Kantorenamt im Stift Heiligenkreuz, wohin Rueff im gleichen Jahr als Abt berufen wurde. Amon trat 1587 in das Franziskanerkloster in Wien ein, empfing hier die Priesterweihe, leitete den Chor und verstarb bereits im Juni 1590. ${ }^{14}$

Blasius Amon dankte in der Dedikation seines ersten gedruckten Werkes aus dem Jahr 1582, genannt LIBER SACRATISSIMARUM (QVAS VVLGO INTROITUS APPELLANT) CANTIONUM SACRUM SELECTISSIMUS, Johannes Rueff für seine Gönnerschaft: „Dann stütze ich mich bei ihrer Veröffentlichung [der Introita] auf den Klang und das Ansehen deines Namens, da ich aus Erfahrung weiß, dass Eure Herrlichkeit fast allen Musikern gegenüber die Gesinnungen eines Gönners hegen, und dass dieselben an mir fürwahr die allergrößten Verdienste sich erworben haben [...]. " ${ }^{15}$ Die berufliche und freundschaftliche Beziehung des Komponisten zu Johannes Rueff, zunächst in Zwettl und später in Heiligenkreuz, lässt diesen als Vermittler zwischen Gallus und Amon vermuten. Die Bekanntschaft zwischen Rueff und Gallus ist in der Forschung bereits bekannt. Gallus widmete ihm seinen vierten Band der Messen und dankte im Vorwort für das Wohlwollen und die - vermutlich im Stift Melk - miteinander verbrachte Zeit. ${ }^{16}$

${ }^{12}$ Caecilianus Huigens O.F.M., Fr. Blasius Amon (ca 1560-1590). Sein Leben und seine Werke. Ein Beitrag zur Geschichte der Kirchenmusik in Österreich, Wien, 1914, S. 435. (Universität Wien, Dissertation). Hervorhebung nicht original.

${ }^{13}$ Walter Senn, Zur Lebensgeschichte des Tiroler Komponisten Blasius Amon (um 1560-1590). Mit einem Werkverzeichnis. In: Veröffentlichungen des Tiroler Landesmuseum Ferdinandeum, Bd. 56, München, Salzburg, Katzbichler, 1976, S. 145-168. Eine praktische Ausgabe der Messe besorgte Wolfgang Fürlinger 1993 beim Musikverlag Alfred Coppenrath, Altötting. Eine weitere Transkription von Thomas Engel befindet sich in elektronischer Form unter: http://www.musikland-tirol.at/html/musikedition/komponisten/amon/opusmissae/missae.html (20. August 2007).

${ }^{14}$ Vgl. Bettina Schwemer / (Arnold Geering), Amon, Ammon, Blasius, Die Musik in Geschichte und Gegenwart. Personenteil 1, 2. Auflage, Kassel [...], Bärenreiter, 1999, Sp. 614-615.

${ }^{15}$ Blasius Amon, Widmung an Johannes Rueff, LIBER SACRATISSIMARUM [...], Wien, Stephan Creuzer, 1582, (RISM A/I: A 940); deutsch von C. Huigens, op. cit., S. 150 f.; vgl. auch lateinischen Originaltext, ebd., S. 144.

${ }^{16}$ Johannes Rueff war zwischen 1572 und 1580 in Melk tätig. 
Während Gallus von den Förderungen, Ermutigungen und einem stets freundschaftlichen Zusprechen vonseiten Rueffs berichtet, ${ }^{17}$ dürfen wir annehmen, dass dieser auf gleiche Art auch seinen Kantor Blasius Amon im Komponieren bestärkte. Es liegt nahe, dass er ihn auch mit dem Werk von Gallus bekannt machte. Ob sich allerdings die Komponisten persönlich begegneten und zwischen beiden ein direkter Austausch stattfand, konnte bisher nicht nachgewiesen werden.

Die MISSAE QVATVOR, VOCIBUS QVATERNIS IN DIVINO DEI [...] von Blasius Amon wurden 1588 mit einem kaiserlichen Privileg bei Michael Apffl in Wien gedruckt (RISM A/I: A 941). Die dem Abt des Zisterzienserstiftes Lilienfeld Laurentius Reyß gewidmete Sammlung enthält vier vierstimmige Ordinariumsvertonungen und eine Missa pro defunctis. An dritter Stelle steht darin die Missa super Surge propera. Vom genannten Druck sind lediglich zwei unvollständige Exemplare erhalten, das eine in der Universitätsbibliothek Uppsala und das zweite in der Stadtbibliothek Wien (Musiksammlung). Verbreiteter ist dagegen eine neue Auflage des Werkes, die 1591 posthum in der Druckwerkstatt von Adam Berg in München erfolgte. Berg nahm das Werk in seine Reihe Patrocinium musices auf, veränderte den Titel in MISSAE CVM BREVES TVM QVATVOR VOCVM laudatissime concinnatae (RISM A/I: A 942) und höchstwahrscheinlich aufgrund des kaiserlichen Privilegs auch die gesamte Anlage. Die Messen sind hier in einem großformatigen Chorbuch in Folio erschienen. Der Drucker pries in der Widmung an Kardinal Andreas, Bischof von Konstanz und Brixen, die ,[...] anerkannt feine Art der Zusammenstellung als auch in allen menschlichen Handlungen erwünschte und lobenswerte Kürze $[\ldots]^{“ 18}$ dieser Messen. Dass Amon, bekanntlich ein Meister der kleinen Form, ${ }^{19}$ auch in seinen Messen Bündigkeit und Kürze anstrebte, ist deutlich erkennbar. Die Länge der Vorlage mit ihren 71 Takten wird in der Missa super Surge propera lediglich vom 108 Takte umfassenden Credo übertroffen.

Die Vorgehensweise Amons in der Bearbeitung der Motette kann anhand des dritten Kyrie veranschaulicht werden (siehe Notenbeispiel 3). Im zehn Takte umfassenden Satz zitiert Amon zunächst, abgesehen von einer Veränderung im Tenor, den Beginn der secunda pars der Motette von Handl (A). Danach greift er in die Satzstruktur ein (B) und vertauscht die Stimmen: im Cantus erklingt das Motiv des Altus, im Tenor das des Bassus und im Bassus die Melodiewendung des Cantus. Die Klausel (C) wurde vom Komponisten hinzugefügt. Der letzte Teil des dritten Kyrie (D) ist als mehrstimmiger Komplex dem abschießenden ersten Vers der secunda pars von Gallus entnommen und in einer rhythmisch veränderter Form eingesetzt worden. Dieses Verfahren, das primär aus der Übernahme, Verkürzung und Modifizierung der Vorlage unter gelegentlicher Hinzufügung von Neuem besteht, wird zum Kompositionsprinzip erhoben und ist auch für alle übrigen Messsätze bestimmend. ${ }^{20}$

\footnotetext{
${ }^{17}$ Edo Škulj, Gallusovi predgovori in drugi dokumenti, Ljubljana, Družina, 1991, S. 44.

${ }^{18}$ Adam Berg, Vorrede zu MISSAE CVM BREVES TVM QVATVOR VOCVM [...], München, Adam Berg, 1591; deutsch von C. Huigens, op. cit., S. 226.

${ }^{19}$ W. Senn, op. cit., S. 165.

${ }^{20}$ In Rahmen von diesem Beitrag kann nur auf die wesentlichsten Charakteristika der Messe von Blasius Amon hingewiesen werden. Eine eingehende Analyse des Werkes steht noch bevor.
} 


\section{Notenbeispiel 3}

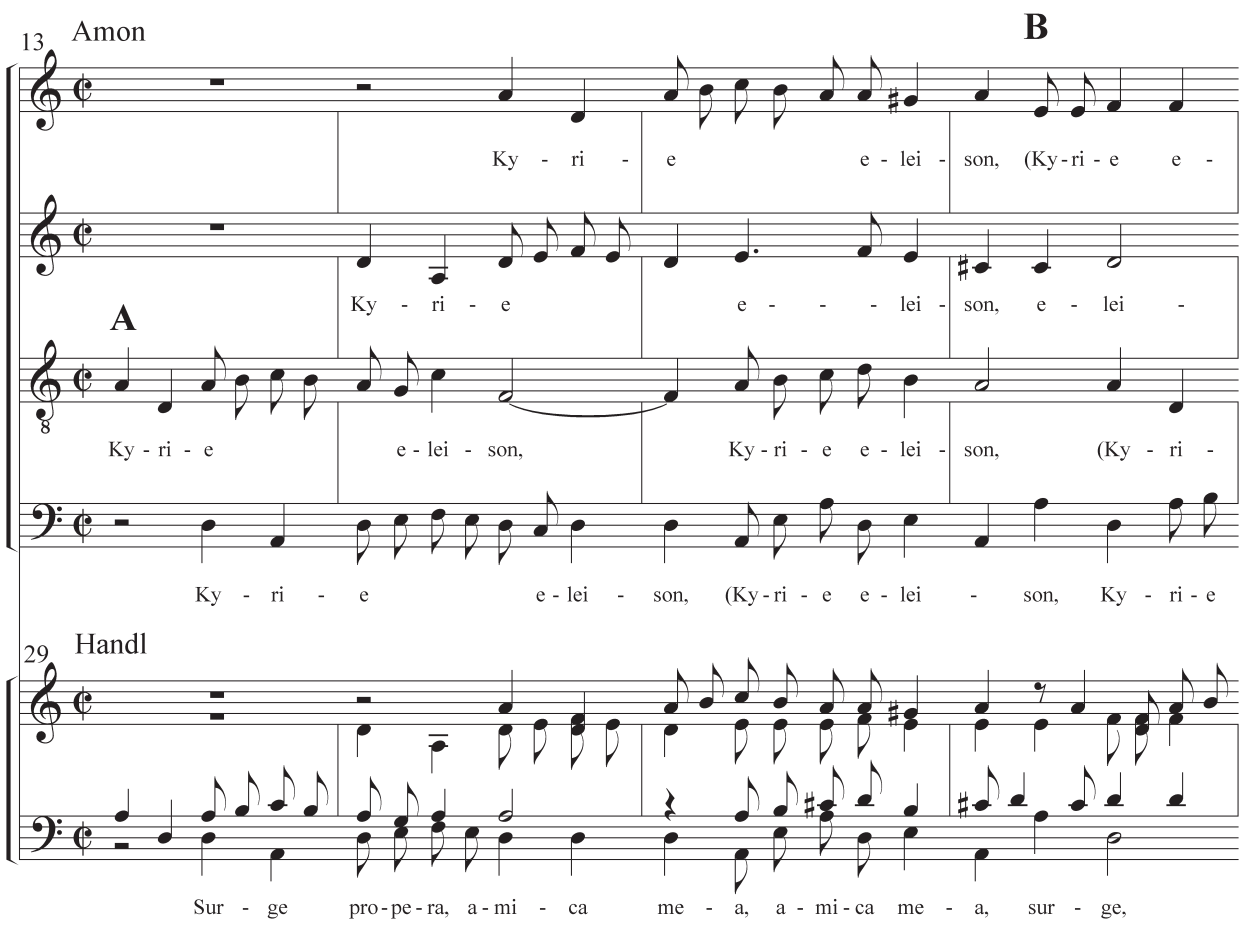

Blasius Amon, Missa super Surge propera, Kyrie, T. 13-20 (oben), im Vergleich mit dem Vokalsatz von Jacob Handl, T. 29-36 (unten). ${ }^{21}$

Als einer der wenigen Theoretiker des späten 16. Jahrhunderts, die genaue Angaben zum Aufbau einer so genannten Parodiemesse ausformuliert haben, gilt Pietro Cerone. Obwohl sein 1613 in spanischer Sprache publiziertes Traktat El melopeo y maestro ${ }^{22}$ im deutschsprachigen Raum wohl wenig Nachklang fand, dürfen wir annehmen, dass seine Beschreibungen die mehr oder weniger gängige Praxis des späten 16. Jahrhunderts spiegeln. Ein Vergleich der Ausführungen Cerones mit der Messe von Blasius Amon ist daher durchaus lohnenswert.

Pietro Cerone fordert vorerst, die Anfänge aller Hauptsätze der Messe übereinstimmend mit dem gleichen motivischen Material zu gestalten, das dem Beginn der Vorlage entnommen sein muss. Die Satzanfänge sollten jedoch Mannigfaltigkeit in der Bearbeitung des musikalischen Materials aufweisen. Blasius Amon hält sich an diese Vorgabe nur

bei der Gestaltung der Anfänge von Kyrie, Gloria und Sanctus, die auf dem Beginn der

${ }^{21}$ Transkribiert (brevis $=$ ganze Note) nach der Ausgabe von Adam Berg, München, 1591, Exemplar der Österreichischen Nationalbibliothek, Musiksammlung, Signatur SA.25.Aa.6 Mus 27.

22 Pietro Cerone, El melopeo y maestro, Buch XII, [Neapel 1613]; übersetzt ins Englische von Oliver Strunk, Source Readings in Music History, From Classical Antiquity through the Romantic Era, New York, W. W. Norton \& Company, 1950, S. 265-268. 
Notenbeispiel 3

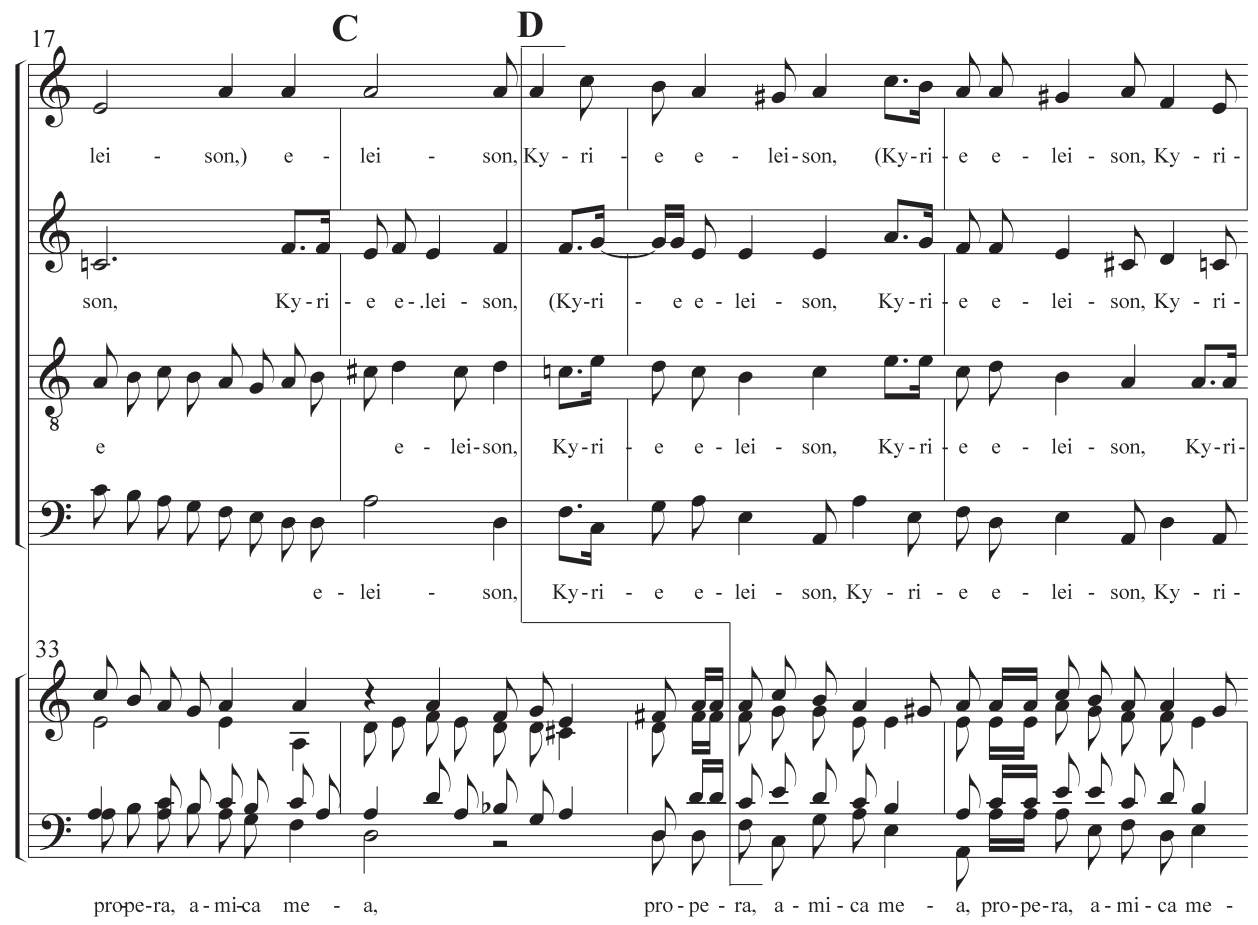

Motette Surge, propera basieren. Der Anfang des Credo ist dem Motiv des ersten Verses der secunda pars entliehen, während das Motiv des Agnus Dei vom dritten Vers der prima pars („Flores apparuerunt“) stammt. Cerone möchte ferner die Hauptsätze ebenso mit der Bearbeitung eines identischen Motivs beendet wissen. Amon befolgte diese Regel ziemlich strikt. In den Schlussgruppen der Sätze tritt jeweils die stufenweise absteigende Quarte auf. Für das Christe erlaubt Cerone ein Motiv aus der Mitte des Modells zu wählen. Bei Amon ist das wiederum die absteigende Quarte, übernommen aus dem zweiten Vers der prima pars (,imber abiit et recensit“). Sein Christe bildet zu den polyphonen $K y$ rie-Teilen einen kontrastierenden homophonen Abschnitt. Die der Vorlage entnommene Motivgruppe musste zu diesem Zweck allerdings rhythmisch geglättet und geringfügig modifiziert werden. Cerone bemerkte, dass der Komponist das dritte Kyrie, sowie das zweite und dritte Agnus Dei frei erfinden oder auf ein Nebenmotiv aus der Vorlage zurückgreifen soll. Amon verwendete im dritten Kyrie ähnlich wie beim Credo den Quintfall aus der secunda pars der Motette (siehe Notenbeispiel 3), sein Agnus Dei ist jedoch ohne Angaben zur Aufführung des zweiten und dritten Agnus bloß einmal durchkomponiert. Die Textstellen Jesu Christe im Gloria, sowie Et incarnatus und Crucifixus im Credo sollten laut Pietro Cerone mit langen Noten und ohne Imitationen gesetzt werden. Während das Jesu Christe von Amon zwar mit langen Noten deklamiert wird, schenkt er die- 
ser Textstelle ansonsten keine besondere Beachtung. Die Regel wird jedoch eindeutig im homophonen Et incarnatus erfüllt, dessen musikalisches Material auch nicht der Vorlage entnommen ist. Im Widerspruch mit der Anweisung Cerones steht das imitativ gesetzte Crucifixus, das erneut auf dem Quintfallmotiv basiert. Der Satzteil ist entsprechend den Empfehlungen zu drei Stimmen gesetzt. Im weitgehend frei ersonnenen dreistimmigen Benedictus werden die Motive der stufenweise auf- und absteigenden Quarten miteinander kombiniert. Die Motive kommen in der Vorlage von Gallus zwar häufig vor, einer bestimmten Textstelle können sie jedoch nicht ausdrücklich zugeordnet werden.

Die Ausgewogenheit und Abwechslung zwischen dem Übernommenen und aus der Thematik des Modells eigenständig Erschaffenem bei Blasius Amon ist beachtlich. Ein schablonenartiges Vorgehen liegt ihm fern. Amon entnahm der Vorlage nur sorgfältig ausgewählte und als geeignet erachtete Satzgruppen oder charakteristische Motive, die von verschiedensten Stellen der Motette stammen können. Eine gewisse Zurückhaltung vor einer allzu wörtlichen Übernahme macht sich deutlich bemerkbar. Manchen Satzteilen von Gallus wird zwar der Messtext direkt unterlegt, diese Zitate sind allerdings jeweils verkürzt und geringfügig verändert worden. Oft werden aus der Motette auch bloß einzelne Motive übernommen, die wiederum erweitert, vereinfacht oder in ihrem Wesen mannigfaltig verändert wurden. In manchen Teilen entfernt sich Amon auch ziemlich weit von der Vorlage und schafft - vor allem an Kadenzstellen - völlig neu konzipierte Passagen. Stellenweise übernimmt er nur die Kompositionsweise von Gallus, beispielsweise die Stimmführung in Stimmpaaren bei „Et ex Patrem natum“, nicht jedoch die Thematik dieser Stelle.

Nach welchem Prinzip die Motive für die jeweiligen Textsegmente ausgewählt werden, kann nicht eindeutig geklärt werden. Das Bestreben nach bloßer Abwechslung spielte bestimmt eine gewisse Rolle. Bei manchen Stellen ist allerdings auch eine Intention nach Textausdeutung sowie einfache Tonmalerei zu erkennen. So wird beispielsweise für die Worte „,descendit de coelis“ der absteigende Dreiklang aus dem vierten Vers der secunda pars (,sonet vox tua“) gewählt. Auf eine ähnliche Weise wird die Textstelle „Et resurrexit“ mit einem Quintsprung oder „Et ascendit in coelum“ durch die stufenweise aufsteigende Tonfolge aus dem vierten Vers der prima pars („,Tempus putationis“) charakterisiert.

Zusammenfassend kann gesagt werden, dass sich die Missa super Surge propera durch einen großen Abwechslungsreichtum auszeichnet. Die homophonen und polyphonen Abschnitte, syllabischen und melismatischen Textpassagen, sowie zwei- bis vierstimmigen Satzteile kontrastieren miteinander und bilden trotzdem ein Gesamtwerk von beachtenswerter Geschlossenheit. In der Vorgehensweise Amons ist eine derartige Freiheit und künstlerische Souveränität festzustellen, dass diese bisher kaum beachtete Messe unter die hervorragendsten Zeugnisse der Gallus-Rezeption eingeordnet werden muss.

Ob Jacob Handl Gallus die beiden Bearbeitungen der Motette kannte, ist ungewiss. Im Hinterlassenschaftsprotokoll des verstorbenen Komponisten, das (abgesehen von seinen eigenen Drucken) interessanterweise ohnehin nur Musikalien aus den Nürnberger Druckwerkstätten beinhaltet, sind weder die Sammlungen von Kargel und Amon noch 
die von Stephan Schormann aufgelistet. ${ }^{23}$ Dass Gallus auf einer seiner zahlreichen Reisen mit diesen Drucken in Berührung kam ${ }^{24}$ oder dass ihm Johannes Rueff über die Messe von Amon berichtete, ist denkbar, keineswegs jedoch belegt. Anhand dieser Werke dürfen wir jedenfalls auf eine frühe und weite handschriftliche Verbreitung der Motette schließen. Die beiden Bearbeitungen zeugen von der Beliebtheit und Wertschätzung der Musik von Jacob Handl Gallus und sind noch zu den Lebzeiten des Komponisten wertvolle Zeugnisse einer regen Rezeption.

${ }^{23}$ Vgl. E. Škulj, op. cit., S. 141-149.

${ }^{24}$ Ein Exemplar des Lautenbuchs von Sixtus Kargel befand sich beispielsweise in Breslau. Vgl. Emil Bohn, Bibliographie der Musik-Druckwerke bis 1700 welche in der Stadtbibliothek, der Bibliothek des Academischen Instituts fuer Kirchenmusik und der Koeniglichen und UniversitätsBibliothek zu Breslau aufbewahrt werden. Ein Beitrag zur Geschichte der Musik im XV., XVI. und XVII. Jahrhundert, Berlin, Albert Cohn, 1883, S. 225. 


\section{RAZŠIRJENOST MOTETA \\ SURGE, PROPERA, AMICA MEA JACOBUSA HANDLA - GALLUSA \\ TER PREDELAVI SKLADBE SIXTUSA KARGELA IN BLASIUSA AMONA: PRISPEVEK K RECEPCIJI GALLUSOVIH DEL}

\section{Povzetek}

Ob koncu leta 1590 je praški tiskar Georg Nigrin natisnil četrti, zadnji in najobsežnejši del Glasbenega dela (Opus musicum) Jacobusa Handla - Gallusa. Zbirka motetov za praznike svetnikov vključuje dve uglasbitvi druge pesmi po biblični Visoki pesmi, Surge, propera, amica mea. Skladatelj je v obeh delih uporabil sorodno, deloma enako motivno gradivo. Štiriglasni motet Surge, propera je bil že pred objavo nedvomno dolgo časa poznan in razširjen po Evropi. Nekaj mesecev pred Gallusovim avtoriziranim tiskom je skladbo objavil Stephan Schormann v zbirki Suavissimorum modulorum selectissimae cantiones sacrae v Münchnu. Kar štiri leta pred tem pa motet zasledimo v knjigi za lutnjo Lautenbuch viler Newerlesenen fleissiger schöner Lautenstück (1586) škofovskega lutnjarja Sixta Kargla iz Strasbourga. Tudi tirolski skladatelj Blasius Amon je skladbo uporabil kot model za svojo Misso super Surge propera. To delo je bilo objavljeno leta 1588 v zbirki Missae quatuor pri tiskarju Michaelu Apfflu na Dunaju ter 1591 ponatisnjeno pri Adamu Bergu v Münchnu. Pri skladbi Sixta Kargla gre za eno izmed redkih ohranjenih intabulacij Gallusovih del za lutnjo. Žal je delo le bolj ali manj natančen prepis moteta, v katerem pogrešamo več inštrumentalnih figur. Vrstijo se napake v notnem zapisu, obravnavanje kromatičnih tonov je samovoljno in skladba je umetniško manj zanimiva. Povsem drugače in precej bolj suvereno se je motetu približal Blasius Amon. V posameznih mašnih stavkih Amon uporablja zgolj skrbno izbrane dele moteta, ki jih deloma natančno navaja, deloma preoblikuje ter dodaja iz tematike skladbe samostojno komponirane odstavke. Missa super Surge propera sodi med najodličnejše predelave Gallusovih del in Blasius Amon za svojim precej bolj poznanim sodobnikom v ničemer ne zaostaja. Predelavi moteta ne izpričujeta le zgodnje razširjenosti Gallusovega dela, temveč že za časa skladateljevega življenja dokazujeta priljubljenost njegove glasbe po vsej Evropi. 